\title{
ANÁLISIS COMPARATIVO DE LA RESISTENCIA A LA COMPRESIÓN, FLEXIÓN Y TRABAJABILIDAD DEL CONCRETO TRADICIONAL VERSUS UN CONCRETO UTILIZANDO ESCORIA DE ACERO COMO AGREGADO FINO
}

\section{COMPARATIVE ANALYSIS OF THE STRENGTH TO COMPRESSION, FLEXURE AND WORKABILITY OF TRADITIONAL CONCRETE VERSUS A CONCRETE USING STEEL SLAG AS FINE AGGREGATE}

\author{
Armando, Camarena Flores ${ }^{1}$; David, Díaz Garamendi ${ }^{2}$
}

Recibido 06/10/2021: Aprobado: 18/11/2021

DOI: https://doi.org/10.51372/gacetatecnica231.3

\section{RESUMEN}

La presente investigación tiene como objetivo determinar la influencia de la escoria como agregado global en la resistencia a la compresión, flexión y trabajabilidad para un concreto $f^{\prime} c=20594 \mathrm{kPa}$. Se utilizó la escoria de acero en $10 \%, 15 \%$ y $20 \%$ como agregado fino, de esta manera se muestra una alternativa para el uso de materiales reciclados, con el fin de crear un concreto eficiente y económico, así como también amigable con el medio ambiente, donde hay mucha cantidad de escoria de acero, proveniente de talleres y empresas dedicadas a las estructuras metálicas. Esto debido a que utilizan soldadura de acero, con el fin de minimizar la contaminación ambiental, dado que se demostraría que la escoria de acero tendría un buen comportamiento para el desarrollo de un concreto experimental. El método empleado es cuantitativo utilizando como herramienta de recolección de datos paneles de observación de datos, utilizan estadísticas inferenciales de los datos obtenidos para demostrar hipótesis. Se concluyó que las pruebas de rotura, desgaste y elasticidad realizadas a los 7, 14 y 28 días proporcionaron el diseño óptimo a usar el $10 \%$ para el uso de escoria de acero reciclada en los tres objetivos preestablecidos.

Palabras clave: escoria de acero; resistencia a la comprensión; flexión y trabajabilidad

\footnotetext{
${ }^{1}$ Armando, Camarena Flores, ingeniero civil, Universidad Peruana Unión, asistente en constructora Gallo, Perú, Correo: armandocamarena@upeu.edu.pe, ORCID: https://orcid.org/0000-0001-7698-901X

${ }^{2}$ David, Díaz Garamendi, ingeniero civil asesor de la Universidad Peruana Unión, Perú. Correo: daviddiaz@upeu.edu.pe,ORCID: https://orcid.org/0000-0002-1560-5104
} 


\section{ABSTRACT}

The objective of this research is to determine the influence of slag as a global aggregate on compressive strength, bending and workability for a concrete $f^{\prime} c=20594 \mathrm{kPa}$. Steel slag was used in $10 \%, 15 \%$ and $20 \%$ as fine aggregate, in this way an alternative for the use of recycled materials is shown, in order to create an efficient and economical concrete, as well as friendly with the environment, where there is a large amount of steel slag, coming from workshops and companies dedicated to metal structures. This is due to the fact that they use steel welding, in order to minimize environmental contamination, since it would be shown that the steel slag would have a good behavior for the development of an experimental concrete. The method used is quantitative, using data observation panels as a data collection tool, they use inferential statistics of the data obtained to demonstrate hypotheses. It was concluded that the breakage, wear and elasticity tests carried out at 7, 14 and 28 days provided the optimal design to use $10 \%$ for the use of recycled steel slag in the three preestablished objectives.

Keywords: steel slag; compressive strength; bending and workability

\section{INTRODUCCIÓN}

La industria cementera realiza esfuerzos constantes para reducir el costo de producción del cemento Portland y el consumo de materias primas, proteger el medio ambiente y mejorar la calidad de dicho cemento. Uno de ellos es el uso de ciertos materiales de bajo costo para renovar parcialmente el clínker, como por ejemplo son subproductos industriales y residuos agrícolas [1]. En el mundo, el material más utilizado para la construcción es el concreto, tanto por su flexibilidad, resistencia y trabajabilidad como por su resistencia mecánica. Muchos países han desarrollado métodos para mejorar sus propiedades y la investigación sobre el uso de concreto reforzado con fibra de acero, permitiendo una aplicación optimizada en la estructura. Estas fibras se presentan como una alternativa más para el diseño y construcción de estructuras que requieran propiedades especiales. El uso de concreto reforzado de esta manera se ha incrementado en los últimos años en el Perú, desde los pisos rígidos hasta la mejora de la estabilidad del túnel, convirtiéndolo en un material único y más solicitado en el mercado. Asimismo, la fibra de acero está ahora disponible como una alternativa al diseño y construcción de losas industriales, interrumpiendo por completo los métodos convencionales. [2].

De acuerdo al estudio hecho por la Sociedad Pública de Gestión Ambiental IHOBE [3], los mayores residuos generados por estas industrias son las escorias de acero, que es un material fundido formado por las reacciones químicas entre la materia prima, los materiales añadidos al horno y las impurezas oxidadas que se presentan durante el refinado del metal. Además, en este estudio se afirmó que las escorias más usadas y conocidas en el medio de la construcción son las que derivan de la fabricación de hierro y acero, destacándose la de horno de oxígeno 
básico (BOF) y la de horno eléctrico (EHE o EAF en inglés), las cuales una vez trituradas a una granulometría apropiada presentan propiedades físicas adecuadas para su uso como árido. Estos tipos de hornos son los métodos más usados para la fabricación del acero a nivel mundial.

Es importante señalar que existen instituciones que velan por la calidad y buen uso del acero, y del concreto, por ejemplo, la Asociación Latinoamericana de Acero (ALACERO) [4], es la entidad civil sin fines de lucro que reúne a la cadena de valor del acero de América Latina para fomentar los valores de integración regional, innovación tecnológica, excelencia en recursos humanos, seguridad en el trabajo, responsabilidad empresarial y sustentabilidad socio ambiental. Por otro lado, el Instituto Mexicano del Cemento y del Concreto (http://www.imcyc.com/), refiere que el concreto debe tomar en cuenta tres criterios para tener una buena funcionalidad: costo de materiales y construcción; durabilidad y buen comportamiento ambiental.

Hoy en día en frecuente confundir un concreto económico con uno barato, ya que este último puede ser resistente ante sus solicitaciones de carga, pero eso no quiere decir que es durable, que no se deteriora por las aguas o sulfatos, ya que a un largo plazo a través de los mantenimientos el costo se va a incrementar; mientras el económico es un concreto durable y resistente que implica un costo inicial mayor en su construcción, pero a largo plazo representa ahorros considerables.

\section{DESARROLLO}

Este artículo se basa en la mención del hormigón como base de la investigación, destacando sus propiedades, tipos y composición. Durante el desarrollo, se integró conceptos relacionados con la escoria de acero, así como, el concreto como un material formado a partir de una mezcla, con determinadas propiedades, de cemento, agua, agregados y aditivos opcionales, que inicialmente tiene una estructura plástica y moldeada y que con el tiempo adquiere una consistencia rígida con sus propiedades aislantes y resistentes, que lo convierten en un material ideal para la construcción. Para el desarrollo de la investigación, se realizó una revisión sistemática en bibliotecas electrónicas, Google académico, revistas indexadas, tesis realizadas, artículos y libros personales de construcción, con la finalidad de tener un panorama más amplio sobre la problemática. Las palabras claves utilizadas fueron: estudio y desarrollo de un diseño de mezcla, la norma ACI 211, y escoria de acero parcialmente como agregado fino. 
Las escorias son subproducto de la fundición de la mena para purificar los metales, se les consideran como agregado de óxidos metálicos; suele utilizarse como mecanismo de eliminación de residuos en la fundición del metal, también sirve para el control de temperatura mientras se realiza la fundición además de minimizar la re oxidación del metal líquido. Normalmente se vuelve a procesar para separar los metales que puedan contener, y así pueden ser utilizado como balasto para el ferrocarril y como fertilizante.

\section{METODOLOGÍA}

Teniendo en cuenta el tipo y diseño de la investigación y las características de las variables en estudio, se utiliza el enfoque cuantitativo, utilizando el análisis estadístico inferencial mediante los ensayos de compresión y flexión. La investigación se realizó para obtener el mejoramiento de las propiedades mecánicas utilizando la escoria de acero parcialmente como agregado fino, ubicado en la ciudad de Lima, Perú, porque el material en mención abunda por ese sector debido a las fábricas de acero. La población dependió de una investigación cuasi experimental, por lo que estuvo constituida por probetas cilíndricas de concreto, con el objetivo de mejorar el diseño de mezcla tradicional agregándole escoria de acero, utilizado parcialmente como agregado fino, con porcentajes propuestos de escoria de $10 \%, 15 \%$ y $20 \%$. El tipo de muestreo fue no probabilístico por conveniencia, en la cual se tomó 15 probetas realizadas de manera convencional, y 45 probetas que contuvieron escoria de acero como agregado global del diseño de mezcla:

- Probetas de concreto convencional para ensayo de compresión: 3 probetas de concreto ensayadas para los días 7,14 y 28

- Probetas de concreto con incorporación de escorias de acero $10 \%, 15 \%$ y $20 \%$ para ensayo de compresión: 3 probetas de concreto ensayadas para los días 7 días, 14 y 28

Por lo tanto, se tiene como muestra a 36 probetas para el ensayo de la compresión.

- Probetas de concreto convencional para ensayo de flexión: 2 probetas de concreto ensayadas para los días 7,14 y 28

- Probetas de concreto con incorporación de escoria de acero para ensayo de flexión: 2 probetas de concreto ensayadas para los días 7, 14 y 28 .

Por lo tanto, se tiene como muestra a 24 probetas para el ensayo de la flexión.

De acuerdo a las características de la presente investigación y los enfoques desarrollados, la técnica que se utilizó fue recolección de datos obtenidos de los ensayos de compresión y 
ANÁLISIS COMPARATIVO DE LA RESISTENCIA A LA COMPRESION, FLEXION Y TRABAJABILIDAD DEL CONCRETO TRADICIONAL VERSUS UN CONCRETO UTILIZANDO ESCORIA DE ACERO COMO AGREGADO FINO

flexión en los laboratorios con certificado. Los ensayos de compresión, flexión y trabajabilidad se realizaron solamente en los laboratorios que estaban acreditados y con documentación en regla, tal como MATESTLAB S.A.C. El proceso de validación se hizo según los certificados de los ensayos realizados, y mediante la firma de ingenieros civiles colegiados habilitados que avalaron los procesos antes mencionados.

\subsection{Propuesta}

Para determinar la influencia de la escoria parcialmente como agregado fino en la resistencia a la compresión, para un concreto de $f^{\prime} \mathrm{c}=20594 \mathrm{kPa}$ se propusieron las siguientes hipótesis:

- Hipótesis Nula (Ho): No existe diferencias significativas entre las medias de las resistencias a la compresión para un concreto f'c $=20594 \mathrm{kPa}$ tradicional y un concreto utilizando escoria parcialmente como agregado fino.

- Hipótesis Alterna (Ha): Si existe diferencias significativas entre las medias de las resistencias a la compresión para un concreto $\mathrm{f}^{\prime} \mathrm{c}=20594 \mathrm{kPa}$ tradicional y un concreto utilizando escoria parcialmente como agregado fino.

24 Si el p-valor (sig) o significancia de la prueba es menor a $\alpha$ : se rechaza la hipótesis nula (Ho) y se acepta la hipótesis alterna o del investigador (Ha). De ser lo contrario, se acepta Ho. Se tomó el valor de significancia $\alpha=0,05$.

Para determinar la influencia de la escoria parcialmente como agregado fino en la resistencia a la flexión para un concreto de $f^{\prime} \mathrm{c}=20594 \mathrm{kPa}$ se tienen las siguientes hipótesis:

- Hipótesis Nula (Ho): No existe diferencias significativas entre las medias de las resistencias a la flexión para un concreto $\mathrm{f}^{\prime} \mathrm{c}=20594 \mathrm{kPa}$ tradicional y un concreto utilizando escoria parcialmente como agregado fino.

- Hipótesis Alterna (Ha): Si existe diferencias significativas entre las medias de las resistencias a la flexión para un concreto $\mathrm{f}^{\prime} \mathrm{c}=20594 \mathrm{kPa}$ tradicional y un concreto utilizando escoria parcialmente como agregado fino.

Si el p-valor (sig) $<\alpha$ se rechaza la hipótesis nula (Ho) y se acepta la hipótesis alterna o del investigador (Ha). Si, sig $>\alpha$ entonces se acepta Ho. Donde $\alpha$ es el nivel de significancia y representa el \% de error que estamos dispuesto a asumir. Se tomó el valor de significancia $\alpha=$ 0,05 . 
Para determinar la influencia de la escoria parcialmente como agregado fino en la trabajabilidad para un concreto de $f^{\prime} \mathrm{c}=20594 \mathrm{kPa}$ se tienen las siguientes hipótesis:

- Hipótesis Nula (Ho): No existe interacción significativa entre el tratamiento y el tiempo del mismo que influya en la mejora de la trabajabilidad

- Hipótesis Alterna (Ha): Si existe interacción significativa entre el tratamiento y el tiempo del mismo que influya en la mejora de la trabajabilidad

Si el p-valor (sig) o significancia de la prueba es menor a $\alpha$ entonces se rechaza la hipótesis nula (Ho) y se acepta la hipótesis alterna o del investigador (Ha). De lo contrario se acepta (Ha). Donde $\alpha$ es el nivel de significancia y representa el \% de error que se está dispuesto a asumir. Se tomará el valor de significancia $\alpha=0,05$.

\section{RESULTADOS}

\subsection{Análisis Inferencial para la Resistencia a la Compresión}

A continuación se presentan los resultados del análisis inferencial para la resistencia a la compresión a los 7 y 14 días, reflejado en la Tabla 1 y Tabla 2 respectivamente.

Tabla 1. Resultados de los ensayos a compresión a los 7 días. Fuente: los autores

\begin{tabular}{cc}
\hline Tipo de diseño & Esfuerzo (kPa) \\
\hline & 16400 \\
Diseño patrón optimizado $f^{\prime} c=20594 \mathrm{kPa}$ & 15980 \\
& 16080 \\
Diseño patrón optimizado con $10 \%$ escoria & 16769 \\
de acero $f^{\prime} c=20594 \mathrm{kPa}$ & 17152 \\
& 16867 \\
Diseño patrón optimizado con $15 \%$ escoria & 14710 \\
de acero $f^{\prime} c=20594 \mathrm{kPa}$ & 14514 \\
& 14416 \\
Diseño patrón optimizado con $20 \%$ escoria & 13043 \\
de acero $f^{\prime} c=20594 \mathrm{kPa}$ & 13337 \\
& 13337 \\
\hline
\end{tabular}

Tabla 2. Resultados de los ensayos a compresión a los 14 días. Fuente los autores

\begin{tabular}{cc}
\hline Tipo de diseño & Esfuerzo (kPa) \\
\hline & 20202 \\
Diseño patrón optimizado $f^{\prime} c=20594 \mathrm{kPa}$ & 20006 \\
& 20006 \\
Diseño patrón optimizado con $10 \%$ escoria & 20490 \\
de acero $f^{\prime} c=20594 \mathrm{kPa}$ & 20490 \\
& 20490 \\
\hline
\end{tabular}

Revista Gaceta Técnica. Artículo de Investigación. 23(1), 20-34, enero-junio, 2022 


$\begin{array}{cc} & 18829 \\ \text { Diseño patrón optimizado con } 15 \% \text { escoria } & 18731 \\ \text { de acero } f^{\prime} c=20594 \mathrm{kPa} & 18633 \\ & 18633 \\ & 16377 \\ & 16769 \\ \text { Diseño patrón optimizado con } 20 \% \text { escoria } & 16966 \\ \text { de acero } f^{\prime} c=20594 \mathrm{kPa} & 17162\end{array}$

Los resultados de la prueba de Kruskal Wallis, se muestran en la Tabla 3:

Tabla 3. Resultados de la prueba de Kruskal Wallis. Fuente los autores

\begin{tabular}{cc}
\hline \multicolumn{2}{c}{ Estadísticos de prueba $^{\mathbf{a}, \mathbf{b}}$} \\
\hline Compresión a 14 días \\
H de Kruskal-Wallis & 14,092 \\
gl & 3,000 \\
Sig. asintótica & 0,003 \\
\hline
\end{tabular}

Como el $\mathrm{p}$ valor sig asintótica de la prueba $\mathrm{H}$ de Kruskal Wallis es igual a 0,003 y menor a 0,05, se rechaza la hipótesis nula y se acepta la hipótesis del investigador. Se concluye estadísticamente con un nivel de significancia del 5\%, que existe diferencias específicas entre las medias de las resistencias a la compresión del diseño tradicional, y al menos uno del diseño experimental al añadir escoria de acero parcialmente como agregado fino para un concreto de $f^{\prime} c=20594 \mathrm{kPa}$ a una edad de 14 días.

De igual manera, se muestran los resultados del análisis inferencial para la resistencia a la compresión a los 28 días reflejado en la Tabla 4.

Tabla 4. Resultados de los ensayos a compresión a los 28 días. Fuente los autores

\begin{tabular}{cc}
\hline Tipo de diseño & Esfuerzo (kPa) \\
\hline & 25399 \\
Diseño patrón optimizado $f^{\prime} c=20594 \mathrm{kPa}$ & 24713 \\
& 25105 \\
Diseño patrón optimizado con $10 \%$ escoria de & 2653 \\
acero $f^{\prime} c=20594 \mathrm{kPa}$ & 25574 \\
& 26576 \\
& \\
Diseño patrón optimizado con $15 \%$ escoria de & 22849 \\
acero $f^{\prime} c=20594 \mathrm{kPa}$ & 22849 \\
& 23144 \\
& \\
Diseño patrón optimizado con $20 \%$ escoria de & 19515 \\
acero $f^{\prime} c=20594 \mathrm{kPa}$ & 20006 \\
& 19515 \\
\hline
\end{tabular}

Los resultados de la prueba de Kruskal Wallis, se muestran en la Tabla 5: 
Armando, Camarena Flores; David, Diaz, Garamendi

Tabla 5. Resultados de la prueba de Kruskal Wallis. Fuente: los autores

\begin{tabular}{cc}
\hline \multicolumn{2}{c}{ Estadísticos de prueba $^{\mathbf{a}, \mathbf{b}}$} \\
\hline & Compresión a 28 días $^{2}$ \\
H de Kruskal-Wallis & 14,264 \\
Gl & 3,000 \\
Sig. Asintótica & 0,003 \\
\hline
\end{tabular}

Como se aprecia en la tabla 5, el nivel de significancia obtenido en la prueba de $\mathrm{H}$ de Kruskal Wallis es menor a $0.05(\mathrm{Sig} .=0,003)$ por tal motivo se rechaza la hipótesis nula y se acepta la hipótesis del investigador. Se concluye estadísticamente con un nivel de significancia del 5\%, que existe diferencias significativas entre las medias de las resistencias a la compresión del diseño tradicional y al menos uno del diseño experimental al añadir escoria de acero parcialmente como agregado fino para un concreto de f'c $=20594 \mathrm{kPa}$ a una edad de 28 días.

En la Tabla 6 se muestran los resultados de la prueba Post hoc T3-Dunnet:

Tabla 6. Prueba Post hoc T3 - Dunnet. Fuente: los autores

\begin{tabular}{|c|c|c|c|c|c|c|}
\hline \multicolumn{7}{|c|}{ Comparaciones múltiples } \\
\hline \multicolumn{7}{|c|}{ Variable dependiente: Compresión a 28 días } \\
\hline \multicolumn{7}{|c|}{ T3 Dunnett } \\
\hline \multirow{2}{*}{$\begin{array}{c}\text { (I) FACTORES O } \\
\text { TRATAMIENTO } \\
\mathrm{S}\end{array}$} & \multirow{2}{*}{$\begin{array}{c}\text { (J) FACTORES O } \\
\text { TRATAMIENTO } \\
\mathrm{S}\end{array}$} & \multirow{2}{*}{$\begin{array}{c}\text { Diferencia } \\
\text { de medias } \\
\text { (I-J) }\end{array}$} & \multirow{2}{*}{ Desv. Error } & \multirow[b]{2}{*}{ Sig. } & \multicolumn{2}{|c|}{$\begin{array}{l}\text { Intervalo de confianza al } \\
\qquad 95 \% \\
\end{array}$} \\
\hline & & & & & $\begin{array}{l}\text { Límite } \\
\text { inferior }\end{array}$ & $\begin{array}{l}\text { Límite } \\
\text { superior }\end{array}$ \\
\hline \multirow{3}{*}{$\begin{array}{c}\text { Mezcla } \\
\text { Tradicional }\end{array}$} & 1 al $10 \%$ & $-11,50000^{*}$ & 1,45774 &, 013 & $-18,8159$ & $-4,1841$ \\
\hline & 2 al $15 \%$ & $24,75000^{*}$ & 1,88746 &, 000 & 17,7740 & 31,7260 \\
\hline & 3 al $20 \%$ & $59,25000^{*}$ & 1,86525 &, 000 & 52,3333 & 66,1667 \\
\hline \multirow{3}{*}{$\begin{array}{c}\text { Tratamiento } 1 \text { al } \\
10 \%\end{array}$} & $\begin{array}{c}\text { Mezcla } \\
\text { Tradicional }\end{array}$ & $11,50000^{*}$ & 1,45774 &, 013 & 4,1841 & 18,8159 \\
\hline & 2 al $15 \%$ & $36,25000^{*}$ & 1,25000 &, 000 & 30,0663 & 42,4337 \\
\hline & $\mathrm{T} 3$ al $20 \%$ & $70,75000^{*}$ & 1,21621 &, 000 & 64,7516 & 76,7484 \\
\hline \multirow{3}{*}{$\begin{array}{c}\text { Tratamiento } 2 \text { al } \\
15 \%\end{array}$} & $\begin{array}{c}\text { Mezcla } \\
\text { Tradicional }\end{array}$ & $-24,75000^{*}$ & 1,88746 &, 000 & $-31,7260$ & $-17,7740$ \\
\hline & 1 al $10 \%$ & $-36,25000^{*}$ & 1,25000 &, 000 & $-42,4337$ & $-30,0663$ \\
\hline & 3 al $20 \%$ & $34,50000^{*}$ & 1,70783 &, 000 & 28,2401 & 40,7599 \\
\hline \multirow{3}{*}{$\begin{array}{c}\text { Tratamiento } 3 \text { al } \\
20 \%\end{array}$} & $\begin{array}{c}\text { Mezcla } \\
\text { Tradicional }\end{array}$ & $-59,25000^{*}$ & 1,86525 &, 000 & $-66,1667$ & $-52,3333$ \\
\hline & 1 al $10 \%$ & $-70,75000^{*}$ & 1,21621 &, 000 & $-76,7484$ & $-64,7516$ \\
\hline & 2 al $15 \%$ & $-34,50000^{*}$ & 1,70783 &, 000 & $-40,7599$ & $-28,2401$ \\
\hline
\end{tabular}

Mediante la prueba de T3 de Dunnett se puede observar que al comparar cada tratamiento con respecto a los demás, se identifica que todos tienen diferencias significativas entre sus medias 
ANÁLISIS COMPARATIVO DE LA RESISTENCIA A LA COMPRESION, FLEXION Y TRABAJABILIDAD DEL CONCRETO TRADICIONAL VERSUS UN CONCRETO UTILIZANDO ESCORIA DE ACERO COMO AGREGADO FINO

con respecto al diseño tradicional debido a que los valores de Sig. son iguales a 0,000 y a 0,013, menores a 0,05. Frente a estos resultados, se comprobó que si hay influencia de la escoria parcialmente como agregado fino en la resistencia a la flexión para un concreto $f^{\prime} c=$ $20594 \mathrm{kPa}$.

\subsection{Análisis Inferencial para la Resistencia a la Flexión}

A continuación, se presentan los resultados del análisis inferencial para la resistencia a la flexión a los 7 días reflejado en la Tabla 7 y prueba de post hoc de Tukey en la Tabla 8.

Tabla 7. Resultados de los ensayos a la flexión a los 7 días. Fuente: los autores

\begin{tabular}{cc}
\hline Identificación & Módulo de rotura (kPa) \\
\hline Concreto Patrón & 2844 \\
Concreto Patrón & 2942 \\
Concreto 10 \% de escoria de acero & 2550 \\
Concreto 10 \% de escoria de acero & 2648 \\
Concreto 15 \% de escoria de acero & 2452 \\
Concreto 15 \% de escoria de acero & 2452 \\
Concreto 20 \% de escoria de acero & 2354 \\
Concreto 20\% de escoria de acero & 2354 \\
\hline
\end{tabular}

Tabla 8. Prueba de post hoc de Tukey. Fuente: los autores

\begin{tabular}{|c|c|c|c|c|c|}
\hline \multicolumn{6}{|c|}{ FLEXIÓN A 7 DÍAS } \\
\hline & \multirow{2}{*}{$\begin{array}{c}\text { FACTORES O } \\
\text { TRATAMIENTOS }\end{array}$} & \multirow{2}{*}{$\mathbf{N}$} & \multicolumn{3}{|c|}{ Subconjunto para alfa $=0,05$} \\
\hline & & & 1 & 2 & 3 \\
\hline \multirow{5}{*}{$\begin{array}{l}\text { HSD } \\
\text { Tukey }^{\mathrm{a}}\end{array}$} & 3 al $20 \%$ & 2 & 24,0000 & & \\
\hline & 2 al $15 \%$ & 2 & 25,0000 & 25,0000 & \\
\hline & 1 al $10 \%$ & 2 & & 26,5000 & \\
\hline & Mezcla Tradicional & 2 & & & 29,5000 \\
\hline & Sig. & & 0,322 & 0,124 & 1,000 \\
\hline
\end{tabular}

Mediante la prueba de Tukey se puede visualizar que existe 3 subconjuntos totalmente diferenciados, esto es, entre sus medias para los tratamientos que están en distintos subconjuntos, siendo la del diseño tradicional la que mayor diferencia positiva tiene con respectos a los tres tratamientos.

Seguidamente se presentan los resultados del análisis inferencial para la resistencia a la flexión a los 14 días reflejado en la Tabla 9, y 28 días en la Tabla 10 respectivamente, así como prueba de post hoc de Tukey para 28 días en la Tabla 11. 
Armando, Camarena Flores; David, Diaz, Garamendi

Tabla 9. Resultados de los ensayos a la flexión a los 14 días. Fuente: los autores

\begin{tabular}{cc}
\hline Identificación & Módulo de rotura (kPa) \\
\hline Concreto Patrón & 3334 \\
Concreto Patrón & 3236 \\
Concreto $10 \%$ de escoria de acero & 2942 \\
Concreto $10 \%$ de escoria de acero & 3040 \\
Concreto $15 \%$ de escoria de acero & 2746 \\
Concreto $15 \%$ de escoria de acero & 2746 \\
Concreto $20 \%$ de escoria de acero & 2648 \\
Concreto 20 \% de escoria de acero & 2550 \\
\hline
\end{tabular}

Tabla 10. Resultados de los ensayos a la flexión a los 28 días. Fuente: los autores

\begin{tabular}{cc}
\hline Identificación & Módulo de rotura (kPa) \\
\hline Concreto Patrón & 3923 \\
Concreto Patrón & 4021 \\
Concreto 10 \% de escoria de acero & 4119 \\
Concreto 10 \% de escoria de acero & 4119 \\
Concreto 15 \% de escoria de acero & 3432 \\
Concreto 15 \% de escoria de acero & 3432 \\
Concreto 20 \% de escoria de acero & 3236 \\
Concreto 20 \% de escoria de acero & 3236 \\
\hline
\end{tabular}

Tabla 11. Prueba de post hoc de Tukey. Fuente: los autores

\begin{tabular}{|c|c|c|c|c|c|c|}
\hline \multicolumn{7}{|c|}{ FLEXIÓN A 28 DÍAS } \\
\hline & \multirow{2}{*}{$\begin{array}{c}\text { FACTORES O } \\
\text { TRATAMIENTOS }\end{array}$} & \multirow{2}{*}{$\mathbf{N}$} & \multicolumn{4}{|c|}{ Subconjunto para alfa $=0,05$} \\
\hline & & & 1 & 2 & 3 & 4 \\
\hline \multirow{5}{*}{$\begin{array}{l}\text { HSD } \\
\text { Tukey }^{\mathrm{a}}\end{array}$} & Tratamiento 3 al $20 \%$ & 2 & 33,0000 & & & \\
\hline & Tratamiento 2 al $15 \%$ & 2 & & 35,0000 & & \\
\hline & Mezcla Tradicional & 2 & & & 40,5000 & \\
\hline & Tratamiento 1 al $10 \%$ & 2 & & & & 42,0000 \\
\hline & Sig. & & 1,000 & 1,000 & 1,000 & 1,000 \\
\hline
\end{tabular}

Nota: Se visualizan las medias para los grupos en los subconjuntos homogéneos.

a. Utiliza el tamaño de la muestra de la media armónica $=2,000$.

Mediante la prueba de Tukey se observa que existe 4 subconjuntos totalmente diferenciados, esto es, existe diferencias significativas entre sus medias para los tratamientos que están en distintos subconjuntos, siendo la del tratamiento 1 al añadir el 10\% de escoria de acero la que mayor diferencia positiva tiene con respecto al diseño tradicional y a los otros dos tratamientos.

\subsection{Análisis Inferencial influencia de la Escoria Parcialmente como Agregado Fino}

A continuación, se presentan los resultados de los ensayos de pérdida de trabajabilidad para diseño con 10\%, $15 \%$ y $20 \%$ de escoria reciclada, mostrados en la Tabla 12, 13 y 14 respectivamente. 
ANÁLISIS COMPARATIVO DE LA RESISTENCIA A LA COMPRESION, FLEXION Y TRABAJABILIDAD DEL CONCRETO TRADICIONAL VERSUS UN CONCRETO UTILIZANDO ESCORIA DE ACERO COMO AGREGADO FINO

Tabla 12. Resultados de los ensayos de pérdida de trabajabilidad para diseño con $10 \%$ de escoria reciclada. Fuente: los autores

\begin{tabular}{cccc}
\hline Identificación & $\begin{array}{c}\text { Hora de } \\
\text { ensayo }\end{array}$ & Temperatura & Slump \\
\hline Diseño 10\% E.A & $10: 10 \mathrm{am}$ & $27,1^{\circ}$ & $4^{\prime \prime}$ \\
Diseño 10\% E.A & $10: 40 \mathrm{am}$ & $27,4^{\circ}$ & $31 / 2^{\prime \prime}$ \\
Diseño 10\% E.A & $11: 10 \mathrm{am}$ & $27,9^{\circ}$ & $3^{\prime \prime}$ \\
Diseño 10\% E.A & $11: 40 \mathrm{am}$ & $28,5^{\circ}$ & $21 / 2^{\prime \prime}$ \\
Diseño 10\% E.A & $12: 10 \mathrm{pm}$ & $28,5^{\circ}$ & $1^{\prime \prime}$ \\
Diseño 10\% E.A & $12: 40 \mathrm{pm}$ & --- & --- \\
\hline
\end{tabular}

Tabla 13. Resultados de los ensayos de pérdida de trabajabilidad para diseño con $15 \%$ de escoria reciclada. Fuente: los autores

\begin{tabular}{cccc}
\hline Identificación & $\begin{array}{c}\text { Hora de } \\
\text { ensayo }\end{array}$ & Temperatura & Slump \\
\hline Diseño 15\% E.A & $10: 40 \mathrm{am}$ & $26,9^{\circ}$ & $31 / 2^{\prime \prime}$ \\
Diseño 15\% E.A & $11: 10 \mathrm{am}$ & $27,1^{\circ}$ & $3 "$ \\
Diseño 15\% E.A & $11: 40 \mathrm{am}$ & $27,8^{\circ}$ & $2^{\prime \prime}$ \\
Diseño 15\% E.A & $12: 10 \mathrm{pm}$ & $27,5^{\circ}$ & $11 / 2$ \\
Diseño 15\% E.A & $12: 40 \mathrm{pm}$ & --- & --- \\
Diseño 15\% E.A & $1: 10 \mathrm{pm}$ & --- & --- \\
\hline
\end{tabular}

Tabla 14. Resultados de los ensayos de pérdida de trabajabilidad para diseño con $20 \%$ de escoria reciclada. Fuente: los autores

\begin{tabular}{cccc}
\hline Identificación & $\begin{array}{r}\text { Hora de } \\
\text { ensayo }\end{array}$ & Temperatura & Slump \\
\hline Diseño 20\% E.A & $12: 20 \mathrm{pm}$ & $26,5^{\circ}$ & $3^{\prime \prime}$ \\
Diseño 20\% E.A & $12: 50 \mathrm{pm}$ & $28,4^{\circ}$ & $2^{\prime \prime}$ \\
Diseño 20\% E.A & $1: 20 \mathrm{pm}$ & $28,8^{\circ}$ & $1^{\prime \prime}$ \\
Diseño 20\% E.A & $1: 50 \mathrm{pm}$ & --- & --- \\
Diseño 20\% E.A & $2: 20 \mathrm{pm}$ & --- & --- \\
Diseño 20\% E.A & $2: 50 \mathrm{pm}$ & --- & --- \\
& & & \\
\hline
\end{tabular}

En la Tabla 15 se presentan los resultados de las pruebas de efecto inter - sujetos (ANOVA).

Tabla 15 Pruebas de efecto inter - sujetos (ANOVA). Fuente: los autores

\begin{tabular}{|c|c|c|c|c|c|}
\hline \multicolumn{6}{|c|}{ Pruebas de efectos inter-sujetos } \\
\hline \multicolumn{6}{|c|}{ Variable dependiente: Trabajabilidad slump } \\
\hline Origen & $\begin{array}{c}\text { Tipo III de suma } \\
\text { de cuadrados }\end{array}$ & gl & $\begin{array}{c}\text { Media } \\
\text { cuadrática }\end{array}$ & $\mathbf{F}$ & Sig. \\
\hline Modelo corregido & $100,417^{\mathrm{a}}$ & 23 & 4,366 & 321,222 & 0,000 \\
\hline Intersección & 161,333 & 1 & 161,333 & 11870,018 & 0,000 \\
\hline VAR_TRATAMIENTO & 14,667 & 3 & 4,889 & 359,698 & 0,000 \\
\hline VAR_TIEMPO & 80,979 & 5 & 16,196 & 1191,600 & 0,000 \\
\hline VAR_TRATAMIENTO $*$ VAR_TIEMPO & 4,771 & 15 & 0,318 & 23,401 & 0,000 \\
\hline Error & 0,326 & 24 & 0,014 & & \\
\hline Total & 262,076 & 48 & & & \\
\hline Total corregido & 100,743 & 47 & & & \\
\hline
\end{tabular}

Nota: a. $\mathrm{R}$ al cuadrado $=0,997(\mathrm{R}$ al cuadrado ajustada $=0,994)$

De los resultados se observa que los valores de sig. son iguales a 0,000 y menores a 0,05 para 
cada factor y para la interacción. Esto indica que la hipótesis del investigador fue probada y se rechaza la hipótesis nula. Por tanto, se concluye con 5\% de significancia que existe un efecto en la trabajabilidad debido a cada factor y a su interacción, frente a esto se infiere que dicha trabajabilidad se ve afectada tanto por el tipo de tratamiento y el tiempo de medición.

En la Figura 1 se muestra la interactividad de los dos factores tratamientos y tiempo, se evidencia que la trabajabilidad del diseño tradicional y del tratamiento con el $10 \%$ de escoria de acero es mejor al tratamiento del $15 \%$ y $20 \%$ respectivamente en todo el tiempo de medición. Así también, se concluye que la interactividad del diseño tradicional y el tratamiento 1 del $10 \%$ de escoria de acero, después de una hora y antes de dos horas la trabajabilidad del tratamiento 1 es mejor que la del diseño tradicional.

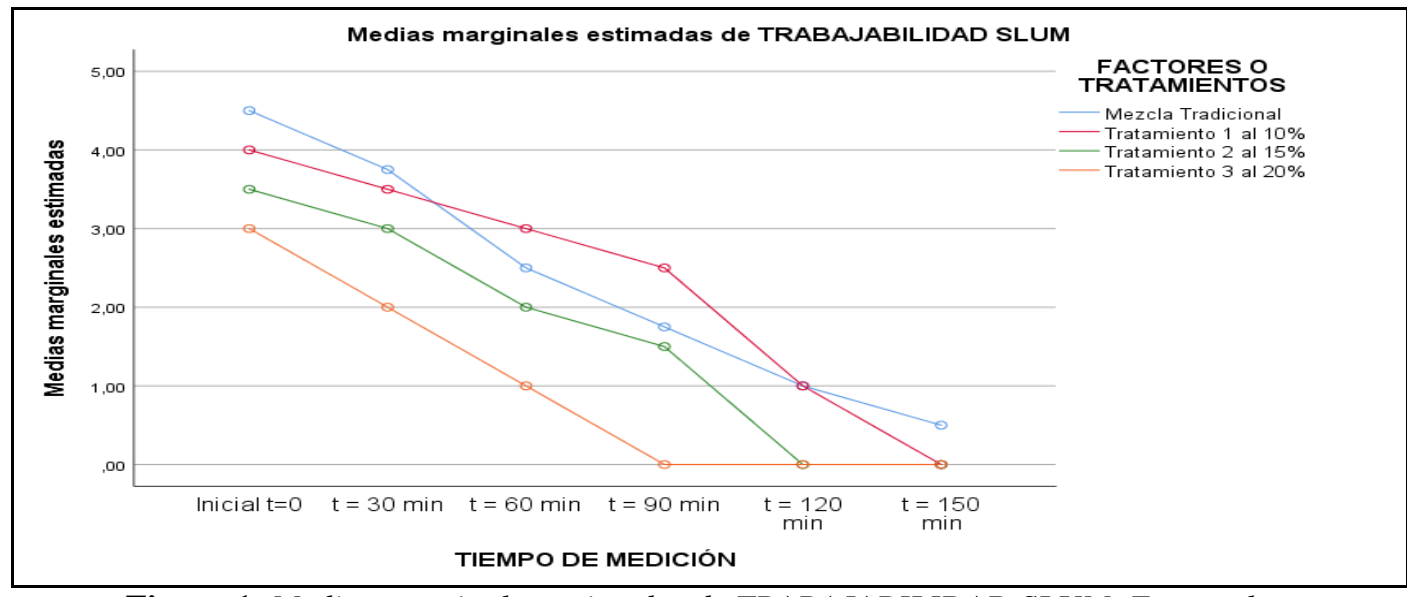

Figura 1. Medias marginales estimadas de TRABAJABILIDAD SLUM. Fuente: los autores

\section{CONCLUSIONES}

Para determinar la influencia de la escoria parcialmente como agregado fino en la resistencia a la compresión para un concreto de $f^{\prime} c=20594 \mathrm{kPa}$, se toman los valores de la resistencia a la compresión para cada grupo y nivel de tratamiento a una edad de 7, 14 y 28 días. Se tiene lo siguiente:

A los 7 días se concluye que con un nivel de significancia del 5\%, al añadir el $10 \%$ de escoria de acero parcialmente como agregado fino mejora significativamente las resistencias a la compresión para un concreto de $f^{\prime} c=20594 \mathrm{kPa}$, mientras que los otros dos tratamientos tuvieron un efecto negativo. A los 14 días se concluye que con un nivel de significancia del $5 \%$, al añadir el $10 \%$ de escoria de acero como agregado global para un concreto de $f^{\prime} c=$ $20594 \mathrm{kPa}$, su media de la resistencia a la compresión permanece invariante respecto al diseño tradicional, mientras que, los otros dos tratamientos tuvieron un efecto negativo. Por último, a los 28 días se concluye que con un nivel de significancia del 5\%, al añadir el 10\% de escoria 
de acero parcialmente como agregado fino para un concreto de $f^{\prime} c=20594 \mathrm{kPa}$, su media de la resistencia a la compresión es significativamente mayor al diseño tradicional entras que, los otros dos tratamientos tuvieron un efecto negativo.

Para determinar la influencia de la escoria parcialmente como agregado fino en la resistencia a la flexión para un concreto de $f^{\prime} c=20594 \mathrm{kPa}$, se toman los valores de la resistencia a la flexión para cada grupo y nivel de tratamiento a una edad de 7, 14 y 28 días. Se observó: a los 7 días se concluye que con un nivel de significancia del 5\%, al añadir el 10\%, 15\% o 20\% de escoria de acero parcialmente como agregado fino no mejora las resistencias a la flexión para un concreto de $f^{\prime} c=20594 \mathrm{kPa}$ de hecho, disminuyen significativamente.

A los 14 días se concluye con un nivel de significancia del 5\% que, al añadir el 10\%, 15\% o $20 \%$ de escoria de acero como agregado global no mejora las resistencias a la flexión para un concreto de $f^{\prime} c=20594 \mathrm{kPa}$, de hecho, disminuyen significativamente, sin embargo, notamos que entre los tres tratamientos la del diseño que se añade el 10\% de escoria de acero, es la que mejor resistencia a la flexión tiene con respecto al añadir el $15 \%$ o $20 \%$.

A los 28 días se concluye con un nivel de significancia del 5\% que, al añadir el 10\%, de escoria de acero parcialmente como agregado fino mejora significativamente la resistencia media a la flexión para un concreto de $f^{\prime} c=20594 \mathrm{kPa}$ con respecto al diseño tradicional, también podemos concluir que al añadir el $15 \%$ o $20 \%$ de escoria de acero, disminuye significativamente la resistencia a la flexión.

Para determinar la influencia de la escoria parcialmente como agregado fino en la trabajabilidad para un concreto de $f^{\prime} \mathrm{c}=20594 \mathrm{kPa}$. Se concluye con 5\% de significancia que existe un efecto en la misma debido a cada factor y a su interacción, esto es que se ve afectada tanto por el tipo de tratamiento y el tiempo de medición. Al añadir el 10\% de escoria de acero la trabajabilidad no varía con respecto al tradicional. La del diseño tradicional y del tratamiento con el $10 \%$ de escoria de acero son mejores al tratamiento del $15 \%$ y $20 \%$ respectivamente en todo el tiempo de medición.

Para dos tratamientos existe un efecto en la trabajabilidad, debido a que sus medias son menores al tradicional y al tratamiento 1 , se concluye a un nivel de significancia del $5 \%$ que disminuye al añadir el $15 \%$ o $20 \%$ de escoria de acero. Entre el diseño tradicional y el tratamiento 1 , no existe diferencia significativa siendo el sig. igual a 1 y mayor a 0.05 , por tanto, se concluye que al añadir el $10 \%$ de escoria de acero la trabajabilidad no varía con respecto al tradicional. La interactividad del diseño tradicional y el tratamiento 1 del $10 \%$ de escoria de acero, podemos concluir que después de una hora y antes de dos horas la del 
tratamiento 1 es mejor que la del diseño tradicional.

\section{REFERENCIAS}

[1] O. A. Díaz, "La evolución de la industria del cemento con énfasis en Latinoamérica", Universidad San Carlos de Guatemala, 2012

[2] A. Sotil y J. Zegarra, “Análisis comparativo del comportamiento del concreto sin refuerzo, concreto reforzado con fibras de acero Wirand ${ }^{\circledR}$ FF3 y concreto reforzado con fibras de acero ....", Disponible en : https://repositorioacademico.upc.edu.pe/handle/10757/581616, 2015

[3] Sociedad Pública de Gestión Ambiental (IHOBE), "Cuentas Anuales e Informe de Gestión 2015", Disponible en: https://www.euskadi.eus/contenidos/informacion/cuentas_anuales_ee_sspp/es_spe_cae/ad juntos/2010_VI_IHOBE.pdf, 2015

[4] Asociación Latinoamericana de Acero (ALACERO), "Asociación Latinoamericana de Acero", Disponible en: https://www.alacero.org, 2020

[5] M. Abdullah y E. Jallo, "Mechanical Properties of Glass Fiber Reinforced Concrete" ALRafdain Eng. J., vol. 20, no. 5, pp. 128-135, doi: 10.33899/rengj.2012.61048, 2012

[6] F. Abanto, "Tecnología del concreto", 3ra Edició. Lima. Perú: Universidad Nacional Mayor de San Marcos, 2018

[7] C. Castiblanco y L. Carrero, "Estudio teórico y experimental del comportamiento del hormigón con materiales no convencionales: Fibras de vidrio y fibras de carbono, sometido a esfuerzos de compresión”, Universidad Católica de Colombia, 2015

[8] A. Montejo, F. Montejo, y A. Montejo, "Tecnología y patología del concreto armado", Bogotá. Colombia: Universidad Católica de Colombia, 2013

[9] M. Olivares Santiago, C. Galán Marín, y J. Roa Fernández, "Los composites. Características y aplicaciones en la edificación," Inf. la Construcción, vol. 54, no. 484, pp. 45-62, doi: 10.3989/ic.2003.v54.i484.568, 2003

[10] M. Salinas, "Elaboración de Expedientes Técnicos", Lima, Perú: Fondo Editorial Instituto de la Construcción y Gerencia (ICG), 2015

[11] Norma ASTM C 31/ C 31M, "Práctica Estándar para Fabricación y Curado de Especímenes de Concreto" USA: ASTM Internacional, 2003

[12] Norma NTP_400.012, "Análisis granulométrico del agregado fino y grueso global" Norma Técnica Peruana, 2002

[13] RNE, "Reglamento Nacional de Edificaciones. E.060 Concreto Armado”, Perú, 2017

[14] ACI 201.2R-07, “Guide to durable concrete”, American Concrete Institute (ACI), 2000

[15] Jo BW, Kim CH, Lim JH, "Characteristics of cement mortar with nano-SiO2 particles" ACI - Mater, 2007

[16] I. Tobón, "Evaluación del Desempeño del Cemento Portland adicionado con Nanoparticulas de Silice", Tesis doctoral, Universidad Nacional de Colombia, Medellín, Colombia, 2011

Revista Gaceta Técnica. Artículo de Investigación. 23(1), 20-34, enero-junio, 2022 
ANÁLISIS COMPARATIVO DE LA RESISTENCIA A LA COMPRESION, FLEXION Y TRABAJABILIDAD DEL CONCRETO TRADICIONAL VERSUS UN CONCRETO UTILIZANDO ESCORIA DE ACERO COMO AGREGADO FINO

[17] E. Huincho Salvatierra, "Concreto de alta Resistencia usando aditivo superplastificante, microsílice y nanosílice con cemento portland tipo I", Tesis de pregrado, Facultad de Ingeniería Civil, Universidad Nacional de Ingeniería, Lima, Perú, 2011 